\title{
Selected CDKN2A and MDM2 polymorphisms in oral cavity cancer
}

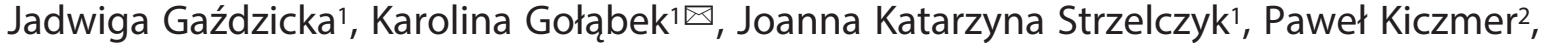 \\ Katarzyna Miśkiewicz-Orczyk³ ${ }^{3}$ Łukasz Krakowczyk4, Zofia Ostrowska and Maciej Misiołek ${ }^{3}$ \\ 'Department of Medical and Molecular Biology, Faculty of Medical Sciences in Zabrze, Medical University of Silesia, Zabrze, Poland; ${ }^{2}$ Depart- \\ ment of Pathomorphology, Faculty of Medical Sciences in Zabrze, Medical University of Silesia, Zabrze, Poland; ${ }^{3}$ Department of Otorhinolaryn- \\ gology and Oncological Laryngology, Faculty of Medical Sciences in Zabrze, Medical University of Silesia in Katowice, Zabrze, Poland; ${ }^{4}$ Clinic of \\ Oncological and Reconstructive Surgery, Maria Sklodowska-Curie Memorial Cancer Center and Institute of Oncology, Gliwice, Poland
}

The head and neck squamous cell carcinoma (HNSCC) is an aggressive human malignancy diagnosed in more than $\mathbf{8 0 0 0 0 0 ~ n e w ~ c a s e s ~ w o r l d w i d e , ~ a n d ~ m o s t l y ~ a r i s e s ~ i n ~}$ the oral cavity, oropharynx, larynx, hypopharynx, and nasopharynx. The study presented here aimed to determine a possible association of rs11515 and rs3088440 gene polymorphisms in the CDKN2A gene (cyclin dependent kinase inhibitor 2A), as well as rs769412 and rs937283 in the MDM2 gene (murine double minute 2), with oral cavity cancer in a sample of Polish population; CDKN2A is crucial in regulating the cell cycle while MDM2 is an oncogene involved in regulating cell proliferation and apoptosis. The study included 95 primary tumor samples following surgical resection from patients, and the control group consisted of 100 healthy individuals. DNA samples were genotyped by employing the $5^{\prime}$ nuclease assay for allelic discrimination using TaqMan SNP Genotyping Assays (Applied Biosystems, USA). There was no significant association between any of the polymorphism (rs11515, rs3088440, rs769412 and rs937283) and the oral cavity cancer risk. We found that the AA homozygotes for rs3088440 were significantly more frequent in the control group (OR=0.046, $p<0.0001)$. In addition, the GG genotype of rs769412 was not found in any group. We found no influence of the examined genotypes on clinicopathological parameters, such as $\mathrm{T}, \mathrm{N}$ and grading values in patients with oral cavity cancer. The results of this study indicate that none of the investigated polymorphisms were associated with the risk of oral cavity cancer in the examined sample of the Polish population.

Key words: head and neck squamous cell carcinoma, oral cavity cancer, $M D M 2$, p16, single nucleotide polymorphism, cancer risk

Received: 03 March, 2020; revised: 27 March, 2020; accepted: 07 April, 2020; available on-line: 07 May, 2020

๑e-mail: kgolabek@sum.edu.pl

*Acknowledgements of Finanacial Support: This work was supported by the Ministry of Science and Higher Education (KNW-121/K/8/O, KNW-1-20/N/7/O).

Abbreviations: HNSCC, head and neck squamous cell carcinoma; OSCC, oral squamous cell carcinoma; HPV, human papillomavirus; SNP, single-nucleotide polymorphisms; CDKN2A, cyclin dependent kinase inhibitor 2A; MDM2, murine double minute 2

\section{INTRODUCTION}

The head and neck squamous cell carcinoma (HNSCC) is an aggressive human malignancy diagnosed in more than 800000 new cases worldwide (Bray et al.,
2018; Canning et al., 2019). Due to high morbidity approximately $50 \%$ of patients die annually (Leemans et al., 2018). The most common sites for the arising of HNSCC are the oral cavity, oropharynx, larynx, hypopharynx, and nasopharynx (Argiris et al., 2008; Marur \& Forastiere, 2016), however the most prevalent subsite is the oral cavity and thus the oral squamous cell carcinoma (OSCC) accounts for about 3\% of all cancers worldwide (Yanamoto et al., 2012; Weckx et al., 2019). The list of risk factors associated with HNSCC includes alcohol and tobacco use, diet, infection with high-risk types of human papillomavirus (HPV), geographical location, habits, as well as the genetic background (Vigneswaran \& Williams, 2014). Dysregulation of numerous genes, such as the tumor suppressor genes, oncogenes, and DNA repair genes, as well as loss of the genome integrity, are widely accepted causes of HNSCC (Gollin, 2014). Single-nucleotide polymorphisms (SNPs) are reported to be important factors in oral cancer susceptibility, and SNPs which are involved in DNA repair, cell cycle control, and carcinogen metabolism may especially be the pivotal factors for this malignancy (Shridhar et al., 2016).

CDKN2A (cyclin dependent kinase inhibitor $2 \mathrm{~A}$ ) is a tumor suppressor gene involved in cell cycle regulation, and it is commonly mutated in HPV-negative patients with the head and neck cancer (Ausoni et al., 2016). CD$K N 2 A$ is located on chromosome 9 and encodes two products, p14-ARF and p16-INK4A (Gallagher et al., 2005). p14-ARF interacts with MDM2 (Murine double minute 2) and as a result leads to ubiquitination of p53 (Gallagher et al., 2005; Ausoni et al., 2016), while p16INK4A blocks phosphorylation of $\mathrm{pRB}$ and negatively regulates the cell cycle by arresting cells in the late G1 phase (Ausoni et al., 2016).

Another important gene in carcinogenesis is the MDM2 oncogene, a negative regulator of p53. MDM2 takes part in regulation of cell proliferation and apoptosis through inhibition of the p53 activity (Denaro et al., 2011), which is performed in the ubiquitination pathway (Yang et al., 2013). Also, MDM2 is a negative regulator of many other molecules, such as pRb, P21, p14 or ribosomal proteins (Rayburn et al., 2005). Up-regulation of MDM2 has been reported in various cancers, and may influence a decreased cellular response to radio- or chemo-therapy (Rayburn et al., 2005). MDM2 polymorphisms may be located in the promoter region, such as rs937283, and in consequence influence the transcription level of that gene (Feng et al., 2016). The purpose of the study presented here was to determine a possible association of rs11515 and rs3088440 polymorphisms in the $p 16$ gene, as well as rs769412 and 
Table 1. Types of polymorphism and primers used

\begin{tabular}{llll}
\hline SNP ID & Codon change & Amino acid change & Context Sequence [VIC/FAM] \\
\hline rs11515 & - & - & TCGGTGACTGATGATCTAAGTTTCC[C/G]GAGGTTCTCAGAGCCTCTCTGGTT \\
\hdashline rs3088440 & - & - & GGTGGGTTGTGGCGGGGGCAGTTGT[A/G]GCCCTGTAGGACCTTCGGTGACTGA \\
\hdashline rs769412 & GAA, GAG & E,E & AACTGGAAAACTCAACACAAGCTGA[A/G]GAGGGCTTGATGTTCCTGATTGTA \\
\hdashline rs937283 & - & - & GTGCCCTGGCCCGGAGAGTGGAATG[A/G]TCCCCGAGGCCCAGGGCGTCGTGCT \\
\hline
\end{tabular}

rs937283 in the MDM2 gene, with HNSCC in a sample of the Polish population.

\section{MATERIALS AND METHODS}

Patients and samples. The study presented here included 95 primary tumor samples obtained from Polish patients following surgical resection at the Department of Otorhinolaryngology and Oncological Laryngology, Faculty of Medical Sciences in Zabrze, Medical University of Silesia, Maria Sklodowska-Curie Memorial Cancer Centre and Institute of Oncology (Gliwice, Poland). All of the tumors collected during surgery were oral cavity cancers (comprising the maxilla, mandible, floor of the mouth, tongue, and cheek, with the highest number being mandible and tongue cases). All samples were quickly frozen at $-80^{\circ} \mathrm{C}$ until DNA extraction. These tumor samples were histologically confirmed by pathologists and were classified as primary tumors. Tumor staging was based on the American Joint Committee on Cancer (AJCC, version 2007) (Brunner et al., 2014; Rodrigues et al., 2014). Patients included in this study had no history of preoperative radio- or chemo-therapy. The control group consisted of 100 healthy individuals. All of the patients and controls were Caucasians who lived in the area of Poland. The study was approved by the Ethics Committee of the Medical University of Silesia (Katowice, Poland; approval no. KNW/022/KB1/49/16), and the Institutional Review Board on Medical Ethics of the Maria Sklodowska-Curie Memorial Cancer Centre and Institute of Oncology in Gliwice (Poland; approval no. $\mathrm{KB} / 493-15 / 08$ and $\mathrm{KB} / 430-47 / 13)$.

DNA extraction. Genomic DNA was extracted from each tumor sample $(20 \mathrm{mg})$ by DNeasy Blood \& Tissue Kits (Qiagen, Hilden, Germany) according to the manufacturer's instructions, after tissue homogenization in a FastPrep ${ }^{\circledR}-24$ instrument using Lysing Matrix A tubes (MP Biomedicals, Solon, CA, USA). In the control group, the DNA was extracted from swabs taken from oral mucous membranes using a Swab-Extract DNA Purification Kit (EURx, Gdansk, Poland) according to the manufacturer's instructions. After that, high-quality cellular DNA was eluted in low salt buffer containing $10 \mathrm{mM}$ Tris- $\mathrm{HCl}, \mathrm{pH}$ 8.5. Qualitative and quantitative analysis of all isolated DNA was performed by spectrophotometry in a Biochrom WPA Biowave DNA UV/Vis Spectrophotometer (Biochrom, Cambridge, UK) according to the manufacturer's instructions.

Single nucleotide polymorphism (SNP) analyses. Genotyping was conducted with a QuantStudio 5 Real-Time PCR System (Applied Biosystems, Foster City, $\mathrm{CA}, \mathrm{USA})$. The reaction solution contained $5 \mu \mathrm{g} \mathrm{DNA}$, $12.5 \mu \mathrm{l}$ TaqMan Genotyping Master Mix (Applied Biosystems, , Foster City, CA, USA), and $1.25 \mu \mathrm{l}$ TaqMan Genotyping Assay (Applied Biosystems, Foster City, CA, USA). The types of polymorphisms and the primers used are displayed in Table 1. SNP calling was read out automatically in QuantStudio Design and Analysis Software v1.5.1 (Applied Biosystems, Foster City, CA, USA).

Statistical analysis. The significance between distributions of genotypes and alleles, T, N and grading were tested using Pearson's $\chi^{2}$ test. Logistic regression modelling was performed to analyse the oral cavity cancer risk, including the examined SNPs, gender, and age. $P$ values $<0.05$ were considered as statistically significant. The statistical software STATISTICA 13 for Windows (TIBCO Software Inc., Palo Alto, CA, USA) was used to perform all analyses.

\section{RESULTS}

\section{Patient characteristics}

A total of 95 patients with oral cavity cancer were included in this study; their clinical parameters are shown in Table 2. The average age was 62 years (range: 15-78 years). There were $69(73 \%)$ men and $26(27 \%)$ women: $65(68 \%)$ of the patients were smokers; $64(67 \%)$ of them reported alcohol consumption; 51 (54\%) were both smokers and alcohol consumers. The control group consisted of 100 healthy individuals. There were 20 smokers $(20 \%)$ and 68 drinkers (68\%), and 20 were consumers of both tobacco and alcohol (20\%). The average age of the controls was 52.92 years (range: 18-69 years). There were $22(22 \%)$ men and $78(78 \%)$ women.

\section{Demographic characteristics and oral cavity cancer risk}

We estimated differences in demographic characteristics, such as gender and age, between cancer patients and controls. There was no significant association of the

Table 2. Clinical parameters of patients with oral cavity cancer

\begin{tabular}{ll}
\hline Clinical parameters & Patients, $\mathrm{n}(\%)$ \\
\hline Histological grading & $16(17)$ \\
\hline G1 (Well differentiated) & $65(68)$ \\
\hline G2 (Moderately differentiated) & $14(15)$ \\
\hline G3 (Poorly differentiated) & $13(14)$ \\
\hline T classification & $23(24)$ \\
\hline T1 & $22(23)$ \\
\hline T2 & $37(39)$ \\
\hline T3 & \\
\hline T4 & $43(45)$ \\
\hline Nodal status & $24(25)$ \\
\hline N0 & $26(27)$ \\
\hline N1 & $2(2)$ \\
\hline N2 & \\
\hline N3 & \\
\hline
\end{tabular}


Table 3. Comparison of genotype distributions in patients with oral cavity cancer and in controls

\begin{tabular}{|c|c|c|c|c|}
\hline Variable & HNSCC (\%) & Control (\%) & OR $(95 \% \mathrm{Cl})$ & $\mathrm{p}$-value \\
\hline \multicolumn{5}{|l|}{ rs11515 } \\
\hline GG & 72 (75.79) & $78(78.00)$ & 1 & - \\
\hline CG & $22(23.16)$ & $20(20.00)$ & $1.19(0.60-2.36)$ & $>0.05$ \\
\hline $\mathrm{CC}$ & $1(1.05)$ & $2(2.00)$ & $0.54(0.05-6.10)$ & $>0.05$ \\
\hline$C G+C C$ & $23(24.21)$ & $22(22.00)$ & $1.13(0.58-2.21)$ & $>0.05$ \\
\hline \multicolumn{5}{|l|}{ rs3088440 } \\
\hline GG & 75 (83.33) & $65(71.43)$ & 1 & - \\
\hline AG & $14(15.56)$ & $6(6.59)$ & $2.02(0.73-5.57)$ & $>0.05$ \\
\hline AA & $1(1.11)$ & $20(21.98)$ & $0.046(0.01-0.33)$ & $<0.0001$ \\
\hline$A G+A A$ & 15 (16.67) & $26(28.57)$ & $0.5(0.24-1.02)$ & $>0.05$ \\
\hline \multicolumn{5}{|l|}{ rs769412 } \\
\hline $\mathrm{AA}$ & $84(89.36)$ & $83(83.84)$ & 1 & - \\
\hline AG & $10(10.64)$ & $16(16.16)$ & $0.62(0.26-1.44)$ & $>0.05$ \\
\hline GG & $0(0.00)$ & $0(0.00)$ & - & - \\
\hline$A G+G G$ & 10 (10.64) & 16 (16.16) & $0.62(0.26-1.44)$ & $>0.05$ \\
\hline \multicolumn{5}{|l|}{ rs937283 } \\
\hline AA & $30(31.91)$ & $30(30.00)$ & 1 & - \\
\hline AG & $50(53.19)$ & $55(55.00)$ & $0.91(0.48-1.71)$ & $>0.05$ \\
\hline GG & $14(14.89)$ & $15(15.00)$ & $0.93(0.38-2.27)$ & $>0.05$ \\
\hline$A G+G G$ & 64 (68.09) & $70(70.00)$ & $0.91(0.50-1.68)$ & $>0.05$ \\
\hline
\end{tabular}

demographic status with the rs1115 and rs3088440 polymorphisms in the CDKN2A gene in these patients, nor with rs769412 and rs937283 in the MDM2 gene. Furthermore, all examined SNPs were also not associated with smoking or the alcohol status.

\section{The rs11515, rs3088440, rs769412, and rs937283 polymorphisms and the oral cavity cancer risk}

Frequencies of the four polymorphisms in the oral cavity cancer cases and cancer-free controls are shown in Table 3. Upon analysis of the CDKN2A polymorphisms, we did not find an association between the rs11515 genotype or allele and the risk of oral cavity cancer. We found that the AA homozygotes for rs3088440 were significantly more frequent in the control group $(\mathrm{OR}=$ $0.046, p<0.0001)$. Differences in the frequency of MDM2 rs769412 and rs937283 genotypes between groups were nonsignificant. Interestingly, we did not find any cases of a GG genotype of rs769412 in either group.

Association between the rs11515, rs3088440, rs769412, and rs937283 polymorphisms and clinicopathological parameters in patients with oral cavity cancer

These genetic polymorphisms were analyzed in the light of the clinical status of each patient, including the lymph node metastasis. There were no significant associations of the clinicopathological parameters, such as the $\mathrm{T}, \mathrm{N}$ and grading values, with the $p 16$ rs11515 and rs3088440 polymorphisms or with the MDM2 rs769412 and rs937283 polymorphisms in these patients (Table 4).

\section{DISCUSSION}

$C D K N 2 A$ is an important tumor suppressor gene involved in cell cycle regulation (Ausoni et al., 2016) and
MDM2 plays a crucial role during carcinogenesis as an oncogene (Denaro et al., 2011). We observed that neither of the $p 16$ polymorphisms, rs11515 or rs3088440, were associated with the risk of oral cavity cancer. However, we found that the AA homozygotes for rs3088440 were significantly more frequent in the control group (OR= $0.046, p<0.0001)$. Our results are similar to those of Pinheiro and coworkers (Pinheiro et al., 2014) who found no significant association between rs11515 and HNSCC, and moreover that none of the genotype variants of this SNP were correlated with clinicopathological parameters in HNSCC patients. In contrast, another study investigating the CDKN2A rs15115 polymorphism for its connection with oral cancer and oral lesions among a North Indian population (Tripathi et al., 2018) reported that the GG genotype decreased the risk for oral disease in comparison to the CC and CG genotypes which were associated with higher risk, and suggested that the $G$ allele may play a protective role against oral lesions and oral cancer.

In studies related to this polymorphism in other cancers, Royds and others (Royds et al., 2016) showed that the CG heterozygotes in rs11515 were significantly more frequent in breast cancer cases and the CG genotype was correlated with an older age and lymph node involvement, in comparison to individuals with the CC genotype. Nevertheless they found that the frequency of rs3088440 in breast cancer patients was not significantly different from controls. Chansaenroj and coworkers (Chansaenroj et al.,2013) investigated the genotype distributions of both CDKN2A polymorphisms: rs11515 and rs3088440, in HPV-infected women and showed that neither was significantly associated with cervical cancer. According to Liu and others (Liu et al., 2017), rs3088440 was not associated with disease-free survival in patients 
Table 4. Association between $p 16$ and MDM2 polymorphisms and $\mathrm{T}, \mathrm{N}$ and grading in patients with oral cavity cancer

\begin{tabular}{|c|c|c|c|c|c|c|c|c|c|c|c|c|c|}
\hline & & & T1 & $\mathrm{T} 2$ & T3 & $\mathrm{T} 4$ & No & N1 & $\mathrm{N} 2$ & N3 & G1 & $\mathrm{G} 2$ & G3 \\
\hline \multirow{6}{*}{ rs11515 } & \multirow{2}{*}{ GG } & $\mathrm{n}$ & 9 & 21 & 17 & 25 & 33 & 16 & 22 & 1 & 14 & 45 & 12 \\
\hline & & $\%$ & 12.50 & 29.17 & 23.61 & 34.72 & 45.83 & 22.22 & 30.56 & 1.39 & 19.44 & 62.50 & 16.67 \\
\hline & \multirow{2}{*}{ CG } & $\mathrm{n}$ & 4 & 2 & 5 & 11 & 10 & 9 & 3 & 0 & 2 & 18 & 2 \\
\hline & & $\%$ & 18.18 & 9.09 & 22.73 & 50.00 & 45.45 & 40.91 & 13.64 & 0.00 & 9.09 & 81.82 & 9.09 \\
\hline & \multirow{2}{*}{$\mathrm{CC}$} & $\mathrm{n}$ & 0 & 0 & 0 & 1 & 0 & 1 & 0 & 0 & 0 & 1 & 0 \\
\hline & & $\%$ & 0 & 0 & 0 & 100 & 0 & 100 & 0 & 0 & 0 & 100 & 0 \\
\hline \multirow[t]{2}{*}{$p$} & & & \multicolumn{4}{|l|}{0.354} & \multicolumn{4}{|l|}{0.297} & \multicolumn{3}{|c|}{0.46149} \\
\hline & & & T1 & $\mathrm{T} 2$ & T3 & $\mathrm{T} 4$ & No & N1 & N2 & N3 & G1 & G2 & G3 \\
\hline \multirow{6}{*}{ rs3088440 } & \multirow{2}{*}{ GG } & $n$ & 11 & 17 & 18 & 29 & 37 & 22 & 15 & 1 & 12 & 52 & 10 \\
\hline & & $\%$ & 14.67 & 22.67 & 24.00 & 38.67 & 49.33 & 29.33 & 20.00 & 1.33 & 16.00 & 69.33 & 13.33 \\
\hline & \multirow{2}{*}{$A G$} & $n$ & 2 & 3 & 4 & 5 & 5 & 3 & 6 & 0 & 4 & 7 & 3 \\
\hline & & $\%$ & 14.29 & 21.43 & 28.57 & 35.71 & 35.71 & 21.43 & 42.86 & 0.00 & 28.57 & 50.00 & 21.43 \\
\hline & \multirow{2}{*}{$\mathrm{AA}$} & $\mathrm{n}$ & 0 & 0 & 0 & 1 & 0 & 1 & 0 & 0 & 0 & 1 & 0 \\
\hline & & $\%$ & 0 & 0 & 0 & 100 & 0 & 100 & 0 & 0 & 0 & 100 & 0 \\
\hline \multirow[t]{2}{*}{$\mathrm{p}$} & & & \multicolumn{4}{|l|}{0.916} & \multicolumn{4}{|l|}{0.418} & \multicolumn{3}{|l|}{0.607} \\
\hline & & & $\mathrm{T} 1$ & $\mathrm{~T} 2$ & T3 & $\mathrm{T} 4$ & No & $\mathrm{N} 1$ & $\mathrm{~N} 2$ & N3 & G1 & $\mathrm{G} 2$ & G3 \\
\hline \multirow{6}{*}{ rs769412 } & \multirow{2}{*}{$\mathrm{AA}$} & $\mathrm{n}$ & 12 & 19 & 18 & 35 & 38 & 24 & 21 & 1 & 13 & 59 & 11 \\
\hline & & $\%$ & 14.29 & 22.62 & 21.43 & 41.67 & 45.24 & 28.57 & 25.00 & 1.19 & 15.48 & 70.24 & 13.10 \\
\hline & \multirow{2}{*}{$A G$} & $\mathrm{n}$ & 1 & 3 & 4 & 2 & 5 & 2 & 3 & 0 & 3 & 5 & 2 \\
\hline & & $\%$ & 10.00 & 30.00 & 40.00 & 20.00 & 50.00 & 20.00 & 30.00 & 0.00 & 30.00 & 50.00 & 20.00 \\
\hline & \multirow{2}{*}{$\mathrm{GG}$} & $\mathrm{n}$ & 0 & 0 & 0 & 0 & 0 & 0 & 0 & 0 & 0 & 0 & 0 \\
\hline & & $\%$ & 0 & 0 & 0 & 0 & 0 & 0 & 0 & 0 & 0 & 0 & 0 \\
\hline \multirow[t]{2}{*}{$\mathrm{p}$} & & & \multicolumn{3}{|l|}{0.439} & & \multicolumn{2}{|l|}{0.894} & & & \multicolumn{2}{|l|}{0.409} & \\
\hline & & & $\mathrm{T} 1$ & $\mathrm{~T} 2$ & $\mathrm{~T} 3$ & $\mathrm{~T} 4$ & No & N1 & $\mathrm{N} 2$ & N3 & G1 & $\mathrm{G} 2$ & G3 \\
\hline \multirow{6}{*}{ rs937283 } & \multirow{2}{*}{$\mathrm{AA}$} & $\mathrm{n}$ & 2 & 10 & 7 & 11 & 17 & 6 & 7 & 0 & 7 & 18 & 4 \\
\hline & & $\%$ & 6.67 & 33.33 & 23.33 & 36.67 & 56.67 & 20.00 & 23.33 & 0.00 & 23.33 & 60.00 & 13.33 \\
\hline & $\Delta G$ & $\mathrm{n}$ & 10 & 11 & 11 & 18 & 19 & 17 & 14 & 0 & 8 & 34 & 8 \\
\hline & $\pi$ & $\%$ & 20.00 & 22.00 & 22.00 & 36.00 & 38.00 & 34.00 & 28.00 & 0.00 & 16.00 & 68.00 & 16.00 \\
\hline & $C C$ & $n$ & 1 & 2 & 3 & 8 & 6 & 3 & 4 & 1 & 1 & 11 & 2 \\
\hline & G & $\%$ & 7.14 & 14.29 & 2143 & 57.14 & 42.86 & 21.43 & 28.57 & 7.14 & 7.14 & 78.57 & 14.29 \\
\hline$p$ & & & 0.427 & & & & 0.320 & & & & 0.688 & & \\
\hline
\end{tabular}

with hepatocellular carcinoma. In another study, no correlation was found between rs3088440 and type 2 diabetes (Xiao et al., 2016).

In our study, we investigated two MDM2 polymorphisms, rs937283 (also known as SNP G2164A) which causes an adenine to guanine change in position 2164 in the promoter region of the gene (Cao et al., 2018), and rs769412. We found no significant difference in the frequency of rs937283 between the oral cavity cancer patients and controls. The relationship between MDM2 polymorphism and squamous cell carcinoma has been rarely investigated; according to Chen and coworkers (Chen et al., 2010) the AG genotype (OR, 2.20), as well as the combined AG/GG genotype (OR, 2.05), was significantly associated with a higher risk of oral squamous cell carcinoma, and further analyses based on OSCC risk in relation to HPV16 L1 led the authors to suggest a connection between the HPV16 status and the MDM2 polymorphism in the head and neck cancer. That study also revealed a significantly increased risk of OSCC in individuals with the AG or GG genotypes and HPV16 L1 seronegativity (OR, 1.85), while in HPV16 L1 seropositive cases it was higher in connection with the AA genotypes (OR, 2.29), and AG or GG genotypes (OR, 6.88) in comparison to cases with $\mathrm{AA}$ genotypes and HPV16 L1 seronegativity. In addition, examination of interaction between the HPV16 L1 seropositivity and the combination of MDM2 rs2279744 and rs937283 polymorphisms showed a borderline significance (Chen et al., 2010). In contrast, another study revealed no association between any genotype of rs937283 with Caucasian HNSCC cases (Yu et al., 2011). Meta-analysis based on 3 studies of rs937283 MDM2 polymorphism showed no significant association between that SNP and the oral cancer susceptibility (Feng et al., 2016). Similarly, Yu and coworkers (Yu et al., 2011) reported a meta-analysis that no rs937283 polymorphisms were associated with the squamous cell carcinoma susceptibility. 
Although no connection between rs937283 and cancer risk was observed in cases of the esophageal squamous cell carcinoma among a Chinese Han population (Yang et al., 2013), Wang and others (Wang et al., 2015) utilized RFLP-PCR to examine the association between MDM2 rs937283 polymorphism and the risk of laryngeal cancer in another Chinese Han population. The analysis of rs937283 showed that the GG homozygotes, as well as individuals with a $G$ allele, had a significantly higher risk of laryngeal cancer when compared to patients with the AA genotype. Furthermore, they observed that rs937283 was correlated with an increased larynx cancer risk among drinkers. The connection between rs937283 polymorphism and susceptibility to a variety of cancers has been analyzed in a few meta-analyses. For liver cancer, Chen and coworkers (Chen et al., 2018) revealed that the $G$ allele and the $G$ variant genotype of rs937283 were significantly associated with a higher risk, while the $G$ allele and GG genotype were connected with an increased susceptibility for breast cancer; no associations were found with the rectal, colon or cervical cancers. However, that result was significant in an Asian population but not in a Caucasian group (Chen et al., 2018). For retinoblastoma, Cao and coworkers (Cao et al., 2018) revealed in a meta-analysis that the rs937283 homozygote (AA vs GG) was connected with a decreased risk when compared to a fixed-effects variant and a recessive model (AA vs AG+GG). In contrast, Jiao and others (Jiao et al., 2016) showed that the AG and GG genotypes of rs937283 were associated with a higher risk and moreover, that patients with the GG genotype and the $G$ allele had poor survival.

The MDM2 rs769412 polymorphism (SNP E354E), which leads to a change of $A$ to $G$ in the 354 codon but does not cause an amino acid change (Ahmad et al., 2015), was not associated with the HNSCC risk in Caucasians in our study. Moreover, we did not observe any GG genotype cases. There have been only a few studies on rs769412 and cancer risk, and only one connected with the head and neck cancer (Wang \& Ma, 2015) where rs769412 was not significantly associated with the larynx cancer risk in a Chinese population, although further exploration revealed that it was associated among drinkers, suggesting that the MDM2 polymorphism may interact with environmental factors and in consequence lead to cancer development. Our study was performed on a Caucasian population, while Wang and others (Wang et al., 2015) reported this association in a Chinese population, possibly reflecting an influence of ethnicity. However, two other reports based on the Chinese populations showed a lack of association between MDM2 polymorphisms and cancer risk; rs769412 was not significantly connected with prostate cancer risk (Xue et al., 2016) and no genotype variants of SNP354 showed a significant connection with retinoblastoma risk (Jiao et al., 2016). On another hand, Pine and others (Pine et al., 2006) found no association between any genotype of rs769412 and lung cancer risk among Caucasian and African-American populations. Ahmad and coworkers (Ahmad et al., 2015) investigated the correlation of rs769412 with smoking-related risk of lung cancer in a Saudi Arabian population and observed that rs769412 $A>G$ was significantly associated with non-smokers. In contrast, Rajamaran and others (Rajamaran et al., 2007) reported that individuals with the $G$ variant of rs769412 $(\mathrm{Ex} 12+162 \mathrm{~A}>\mathrm{G})$ had a significantly decreased risk of glioma.

In conclusion, the results of study presented here indicate that none of the CDKN2A or MDM2 poly- morphisms examined (rs11515, rs3088440, rs769412 or rs937283) were associated with the oral cavity cancer risk in a Polish population.

\section{Acknowledgements}

Not applicable.

\section{Authors' contributions}

$\mathrm{KG}$, research concept and design; KMO, $\mathrm{KK}$, collection of tumor samples; JG, KG, JKS, collection of control group samples; JG, KG, DNA extraction and SNP analyses; JG, KG, PK, data analysis and interpretation; JG, KG, writing of the article; MM, ZO, critical revision of the article; All authors read and approved the final manuscript.

\section{Competing interests}

The authors declare that they have no competing interests.

\section{REFERENCES}

Ahmad D, Bakairy AK, Katheri AM, Tamimi W (2015) MDM2 (RS769412) G>A polymorphism in cigarette smokers: a clue for the susceptibility to smoking and lung cancer risk. Asian Pac J Cancer Prev 16: 4057-4060. https://doi.org/10.7314/APJCP.2015.16.9.4057

Argiris A, Karamouzis MV, Raben D, Ferris RL (2008) Head and neck cancer. Lancet 371: 1695-1709. https://doi.org//10.1016/S01406736(08)60728-X

Ausoni S, Boscolo-Rizzo P, Singh B, Da Mosto MC, Spinato G, Tirelli G, Spinato R, Azzarello G (2016) Targeting cellular and molecular drivers of head and neck squamous cell carcinoma: current options and emerging perspectives. Cancer Metastasis Rev 35: 413-426. https://doi.org//10.1007/s10555-016-9625-1

Bray F, Ferlay J, Soerjomataram I, Siegel RL, Torre LA, Jemal A (2018) Global cancer statistics 2018: GLOBOCAN estimates of incidence and mortality worldwide for 36 cancers in 185 countries. CA Cancer J Clin 68: 394-424. https://doi.org/10.3322/caac.21492

Brunner M, Ng BC, Veness MJ, Clark JR (2014) Comparison of the AJCC $\mathrm{N}$ staging system in mucosal and cutaneous squamous head and neck cancer. Laryngoscope 124: 1598-1602. https://doi. org//10.1002/lary.24549

Canning M, Guo G, Yu M, Myint C, Groves MW, Byrd JK, Cui Y (2019) Heterogeneity of the head and neck squamous cell carcinoma immune landscape and its impact on immunotherapy. Front Cell Dev Biol 7: 52. https://doi.org/10.3389/fcell.2019.00052

Cao Q, Wang Y, Song X, Yang W (2018) Association between MDM2 rs2279744, MDM2 rs937283, and p21 rs1801270 polymorphisms and retinoblastoma susceptibility. Medicine (Baltimore) 97: e13547. https://doi.org//10.1097/MD.0000000000013547

Chansaenroj J, Theamboonlers A, Junyangdikul P, Swangvaree S, Karalak A, Chinchai T, Poovorawan Y (2013) Polymorphisms in TP53 (rs1042522), p16 (rs11515 and rs3088440) and NQO1 (rs1800566) genes in Thai cervical cancer patients with HPV 16 infection. Asian Pac J Cancer Prev 14: 341-346. https://doi.org//10.7314/apjcp.2013.14.1.341

Chen B, Wang J, Wang J, Zhang J, Gu X, Feng X (2018) The study of MDM2 rs937283 variant and cancer susceptibility in a central Chinese population. Technol Cancer Res Treat 17: 1533033818801550. https://doi.org//10.1177/1533033818801550

Chen X, Sturgis EM, Lei D, Dahlstrom K, Wei Q, Li G (2010) Human papillomavirus seropositivity synergizes with MDM2 variants to increase the risk of oral squamous cell carcinoma. Cancer Res 70: 7199-7208. https://doi.org//10.1158/0008-5472.CAN-09-4733

Denaro N, Lo Nigro C, Natoli G, Russi EG, Adamo V, Merlano MC (2011) The role of p53 and MDM2 in head and neck cancer. ISRN Otolaryngol 2011: 931813. https://doi.org//10.5402/2011/931813

Feng C, Zhang E, Xu Z-F, Duan W-Y, Tan X-X, Sun C-F (2016) Association between polymorphisms in MDM2 gene and oral cancer risk: a meta-analysis. Int J Clin Exp Med 9: 15457-15466

Gallagher S, Kefford RF, Rizos H (2005) Enforced expression of p14ARF induces p53-dependent cell cycle arrest but not apoptosis. Cell Cycle 4: 465-472. https://doi.org//10.4161/cc.4.3.1526

Gollin S (2014) Cytogenetic alterations and their molecular genetic correlates in head and neck squamous cell carcinoma: a next generation window to the biology of disease. Genes Chromosomes Cancer 53: 972-990. https://doi.org//10.1002/gcc.22214 
Jiao Y, Jiang Z, Wu Y, Chen X, Xiao X, Yu H (2016) A functional polymorphism (rs937283) in the MDM2 promoter region is associated with poor prognosis of retinoblastoma in Chinese Han population. Sci Rep 6: 31240. https://doi.org//10.1038/srep31240

Leemans CR, Snijders PJF, Brakenhoff RH (2018) The molecular landscape of head and neck cancer. Nat Rev Cancer 18: 269-282. https:// doi.org//10.1038/nrc.2018.11

Liu S, Yang TB, Nan YL, Li AH, Pan DX, Xu Y, Li S, Li T, Zeng XY, Qiu XQ (2017) Genetic variants of cell cycle pathway genes predict disease-free survival of hepatocellular carcinoma. Cancer Med 6: 1512-1522. https://doi.org//10.1002/cam4.1067

Marur S, Forastiere AA (2016) Head and neck squamous cell carcinoma: update on epidemiology, diagnosis, and treatment. Mayo Clin Proc 91: 386-396. https://doi.org//10.1016/j.mayocp.2015.12.017

Pine SR, Mechanic LE, Bowman ED, Welsh JA, Chanock SC, Shields PG, Harris CC (2006) MDM2 SNP309 and SNP354 are not associated with lung cancer risk. Cancer Epidemiol Biomarkers Prev 15: 15591561. https://doi.org//10.1158/1055-9965.EPI-06-0217

Pinheiro UB, de Carvalho Fraga CA, Mendes DC, Marques-Silva L, Farias LC, de Souza MG, Soares MB, Jones KM, Santos SH, de Paula AM, Velásquez-Meléndez G, Guimarães AL (2014) p16 (CDKN2A) SNP rs11515 was not associated with head and neck carcinoma. Tumour Biol 35: 6113-6118. https://doi.org//10.1007/ s13277-014-1809-0

Rajaraman P, Wang SS, Rothman N, Brown MM, Black PM, Fine HA, Loeffler JS, Selker RG, Shapiro WR, Chanock SJ, Inskip PD (2007) Polymorphisms in apoptosis and cell cycle control genes and risk of brain tumors in adults. Cancer Epidemiol Biomarkers Prev 16: 16551661. https://doi.org//10.1158/1055-9965.EPI-07-0314

Rayburn E, Zhang R, He J, Wang H (2005) MDM2 and human malignancies: expression, clinical pathology, prognostic markers, and implications for chemotherapy. Curr Cancer Drug Targets 5: 27-41

Rodrigues PC, Miguel MC, Bagordakis E, Fonseca FP, de Aquino SN, Santos-Silva AR, Lopes MA, Graner E, Salo T, Kowalski LP, Coletta RD (2014) Clinicopathological prognostic factors of oral tongue squamous cell carcinoma: a retrospective study of 202 cases. Int J Oral Maxillofac Surg 43: 795-801. https://doi.org//10.1016/j. ijom.2014.01.014

Royds JA, Pilbrow AP, Ahn A, Morrin HR, Frampton C, Russell IA, Moravec CS, Sweet WE, Tang WH, Currie MJ, Hung NA, Slatter TL (2016) The rs11515 polymorphism is more frequent and associated with aggressive breast tumors with increased ANRIL and decreased p16 (INK4a) expression. Front Oncol 5: 306. https://doi. org//10.3389/fonc.2015.00306
Shridhar K, Aggarwal A, Walia GK, Gulati S, Geetha AV, Prabhakaran D, Dhillon PK, Rajaraman P (2016) Single nucleotide polymorphisms as markers of genetic susceptibility for oral potentially malignant disorders risk: Review of evidence to date. Oral Oncol 61: 146-151. https://doi.org//10.1016/j.oraloncology.2016.08.005

Tripathi P, Singh A, Sanyal S, Yaday S, Nigam K (2018) Association of polymorphism in P16 and myeloperoxidase genes with susceptibility to oral lesions in North Indian population. Meta Gene 17: 88-92. https://doi.org//10.1016/j.mgene.2018.05.005

Vigneswaran N, Williams MD (2014) Epidemiologic trends in head and neck cancer and aids in diagnosis. Oral Maxillofac Surg Clin North Am 26: 123-141. https://doi.org//10.1016/j.coms.2014.01.001

Wang H, Ma K (2015) Association between MDM2 rs769412 and rs937283 polymorphisms with alcohol drinking and laryngeal carcinoma risk. Int J Clin Exp Pathol 8: 7436-7440

Weckx A, Riekert M, Grandoch A, Schick V, Zöller JE, Kreppel M (2019) Time to recurrence and patient survival in recurrent oral squamous cell carcinoma. Oral Oncol 94: 8-13. https://doi. org//10.1016/j.oraloncology.2019.05.002

Xiao S, Zeng X, Fan Y, Su Y, Ma Q, Zhu J, Yao H (2016) Gene polymorphism association with type 2 diabetes and related gene-gene and gene-environment interactions in a Uyghur population. Med $S_{c i}$ Monit 22: 474-487

Xue L, Han X, Liu R, Wang Z, Li H, Chen Q, Zhang P, Wang Z, Chong T (2016) MDM2 and P53 polymorphisms contribute together to the risk and survival of prostate cancer. Oncotarget 7: 3182531831. https://doi.org//10.18632/oncotarget.3923

Yanamoto S, Yamada S, Takahashi H, Yoshitomi I, Kawasaki G, Ikeda H, Minamizato T, Shiraishi T, Fujita S, Ikeda T, Asahina I, Umeda M (2012) Clinicopathological risk factors for local recurrence in oral squamous cell carcinoma. Int J Oral Maxillofac Surg 41: 1195-200. https://doi.org//10.1016/j.ijom.2012.07.011

Yang J, Liu B, Li W, Xiong H, Qiu H, Fu Q, Chen B, Hu G, Yuan $\mathrm{X}$ (2013) Association of p53 and MDM2 polymorphisms with risk of human papillomavirus (HPV)-related esophageal squamous cell carcinoma (ESCC). Cancer Epidemiol 37: 629-633. https://doi. org//10.1016/j.canep.2013.06.001

Yu H, Huang YJ, Liu Z, Wang LE, Li G, Sturgis EM, Johnson DG, Wei Q (2011) Effects of MDM2 promoter polymorphisms and p53 codon 72 polymorphism on risk and age at onset of squamous cell carcinoma of the head and neck. Mol Carcinog 50: 697-706. https:// doi.org//10.1002/mc.20806

Yu H, Li H, Zhang J, Liu G (2016) Influence of MDM2 polymorphisms on squamous cell carcinoma susceptibility: a meta-analysis. Onco Targets Ther 9: 6211-6224 